\title{
Antibiotic-Resistant Neisseria gonorrhoeae Spread Faster with More Treatment, Not More Sexual Partners
}

\author{
Stephanie M. Fingerhuth ${ }^{1,2 *}$, Sebastian Bonhoeffer ${ }^{1}$, Nicola Low ${ }^{2}$, Christian L. Althaus ${ }^{2}$ \\ 1 Institute of Integrative Biology, ETH Zurich, Zurich, Switzerland, 2 Institute of Social and Preventive \\ Medicine (ISPM), University of Bern, Bern, Switzerland \\ * stephanie.fingerhuth@env.ethz.ch
}

\section{Abstract}

The sexually transmitted bacterium Neisseria gonorrhoeae has developed resistance to all antibiotic classes that have been used for treatment and strains resistant to multiple antibiotic classes have evolved. In many countries, there is only one antibiotic remaining for empirical $N$. gonorrhoeae treatment, and antibiotic management to counteract resistance spread is urgently needed. Understanding dynamics and drivers of resistance spread can provide an improved rationale for antibiotic management. In our study, we first used antibiotic resistance surveillance data to estimate the rates at which antibiotic-resistant $N$. gonorrhoeae spread in two host populations, heterosexual men (HetM) and men who have sex with men (MSM). We found higher rates of spread for MSM ( 0.86 to $2.38 \mathrm{y}^{-1}$, mean doubling time: 6 months) compared to HetM ( 0.24 to $0.86 \mathrm{y}^{-1}$, mean doubling time: 16 months). We then developed a dynamic transmission model to reproduce the observed dynamics of $N$. gonorrhoeae transmission in populations of heterosexual men and women (HMW) and MSM. We parameterized the model using sexual behavior data and calibrated it to $N$. gonorrhoeae prevalence and incidence data. In the model, antibiotic-resistant $N$. gonorrhoeae spread with a median rate of $0.88 \mathrm{y}^{-1}$ in HMW and $3.12 \mathrm{y}^{-1}$ in MSM. These rates correspond to median doubling times of 9 (HMW) and 3 (MSM) months. Assuming no fitness costs, the model shows the difference in the host population's treatment rate rather than the difference in the number of sexual partners explains the differential spread of resistance. As higher treatment rates result in faster spread of antibiotic resistance, treatment recommendations for $N$. gonorrhoeae should carefully balance prevention of infection and avoidance of resistance spread.

\section{Author Summary}

More and more infectious disease treatments fail because the causative pathogens are resistant to the drugs used for treatment. For the treatment of Neisseria gonorrhoeae, a sexually transmitted bacterium, drug resistance is a particularly big problem: there is only a single antibiotic left that is recommended for treatment. We aimed to understand how antibioticresistant $N$. gonorrhoeae spread in a sexually active host population and how the spread of 
Competing Interests: The authors have declared that no competing interests exist. resistance can be slowed. From antibiotic resistance surveillance data, we first estimated the rate at which antibiotic-resistant $N$. gonorrhoeae spread. Second, we reproduced the observed dynamics in a mathematical model describing the transmission between hosts. We found that antibiotic-resistant N. gonorrhoeae spread faster in host populations of men who have sex with men than in host populations of heterosexuals. We could attribute the faster spread of resistant pathogens to higher treatment rates. This finding implies that promoting screening to control antibiotic-resistant $N$. gonorrhoeae could in fact accelerate their spread.

\section{Introduction}

Antibiotic-resistant Neisseria gonorrhoeae can evolve and spread rapidly [1]. Resistance is commonly observed against the antibiotic classes penicillin, tetracycline and fluoroquinolones [2-4]. Resistance also emerged against cefixime, an oral third generation cephalosporin, in recent years [2, 3]. Since 2010, cefixime is no longer recommended as first-line treatment [5] following guidelines from the World Health Organization (WHO) that an antibiotic should not be used when more than $5 \%$ of $N$. gonorrhoeae isolates are resistant [6]. Injectable ceftriaxone, in combination with oral azithromycin, is now the last antibiotic remaining as recommended first-line treatment [7]. Although other antibiotics are being tested for their safety and efficacy for $N$. gonorrhoeae treatment [8], no new classes of antibiotics are currently available [4] and management of antibiotics is urgently needed to preserve their efficacy. The current management strategy tries to reduce the overall burden of $N$. gonorrhoeae infection by expanded screening and treatment of hosts $[9,10]$, but the outcome of this strategy for resistance is uncertain. Understanding the drivers of resistance spread and anticipating future resistance trends will provide rationales for antibiotic management and help to improve antibiotic treatment strategies.

Men who have sex with men (MSM) are host populations that have higher levels of antibiotic-resistant $N$. gonorrhoeae than heterosexual host populations [3]. In a study [5] based on the Gonococcal Resistance to Antimicrobials Surveillance Programme (GRASP) in England and Wales, cefixime-resistant $N$. gonorrhoeae were mainly found in MSM until 2011. The authors suggested that cefixime resistance was circulating in a distinct sexual network of highly active MSM and that bridging between MSM and heterosexuals was necessary for subsequent spread among heterosexual hosts. However, cefixime-resistant $N$. gonorrhoeae might have already been spreading undetected in the heterosexual host population.

Mathematical models can help explain the differential observations of antibiotic-resistant N. gonorrhoeae in different host populations. In 1978, Yorke et al. [11] introduced the concept of core groups to model the transmission of $N$. gonorrhoeae. The concept of core groups posits that an infection can only be maintained in a host population if a highly sexually active group of hosts is responsible for a disproportionate amount of transmissions. More recent modeling studies have examined the transmission of antibiotic-resistant $N$. gonorrhoeae. Chan et al. [12] found that prevalence rebounds more quickly to a pre-treatment baseline when treatment is focused on the core group. Xiridou et al. [13] developed a N. gonorrhoeae transmission model to determine the impact of different treatment strategies on the prevalence of N. gonorrhoeae in Dutch MSM. They found that increased treatment rates could increase the spread of resistance, whereas re-treatment could slow it down. Hui et al. [14] used an individual-based $N$. gonorrhoeae transmission model in a heterosexual host population to investigate the effect of a molecular resistance test on the time until 5\% resistance are reported. None of these studies has investigated or explained the differences in the spread of antibiotic-resistant $N$. gonorrhoeae in MSM and heterosexual host populations. 
In this study, we investigated the dynamics and determinants of antibiotic-resistant $N$. gonorrhoeae spread using surveillance data and mathematical modeling. We estimated the rates at which resistance spreads in heterosexual men (HetM) and MSM using surveillance data from the USA and from England and Wales. We then developed a mathematical model of N. gonorrhoeae transmission to reconstruct the observed dynamics of resistance spread. This allowed us to determine the major driver of resistance spread, and to explore the expected rates at which resistance spreads in MSM and heterosexual host populations.

\section{Methods}

Data

Data sources. We used data from the GRASP $[15,16]$ and the Gonococcal Isolate Surveillance Project (GISP) [17]. GRASP is a program of Public Health England (PHE) that monitors antibiotic-resistant $N$. gonorrhoeae in England and Wales. GISP is an equivalent program from the Centers for Disease Control and Prevention (CDC) in the USA. We used Plot Digitizer 2.6.6 [18] to digitize data on the proportion of cefixime- and ciprofloxacin-resistant N. gonorrhoeae from figures that were published online (see S1 and S2 Tables).

Rate of spread. We determined the rate of resistance spread by assuming that the proportion of antibiotic-resistant $N$. gonorrhoeae follows logistic growth. We used the least squares function $n l s$ from the R software environment for statistical computing [19] to fit the following function to the data:

$$
f(t)=\frac{c}{1+a \times \exp (-b t)} .
$$

$f(t)$ represents the proportion of antibiotic-resistant $N$. gonorrhoeae at time $t, c$ is the maximal proportion of antibiotic-resistant $N$. gonorrhoeae (carrying capacity), $a$ is the ratio between antibiotic-sensitive and -resistant N. gonorrhoeae at time 0 , and $b$ is the rate at which the proportion of antibiotic-resistant $N$. gonorrhoeae increases in the initial exponential growth phase. We only used data from the years before the first decline in the proportion of resistant $N$. gonorrhoeae because we were interested in the rate of resistance spread during the initial exponential growth phase and while the antibiotic was still used.

\section{Model}

Transmission model. We developed a mathematical model to describe the spread of antibiotic-resistant $N$. gonorrhoeae in a given host population [12]:

$$
\begin{aligned}
& \dot{S}_{i}=-S_{i} \pi_{i} \sum_{j \in G} \rho_{i j} \beta_{i j} \frac{I_{\text {Sen }_{j}}+I_{\text {Res }_{j}}}{N_{j}}+v\left(I_{\text {Sen }_{i}}+I_{\text {Resi }_{i}}\right)+\tau(1-\mu) I_{\text {Sen }_{i}} \\
& -\alpha S_{i}+\alpha N_{i}-\gamma S_{i}+\gamma N_{i} \sum_{j \in G} S_{j}, \\
& \dot{I}_{S e n_{i}}=S_{i} \pi_{i} \sum_{j \in G} \rho_{i j} \beta_{i j} \frac{I_{S e n_{j}}}{N_{j}}-v I_{S e n_{i}}-\tau I_{S e n_{i}} \\
& -\alpha I_{\operatorname{Sen}_{i}}-\gamma I_{\operatorname{Sen}_{i}}+\gamma N_{i} \sum_{j \in G} I_{\operatorname{Sen}_{j}} \text {, } \\
& \dot{I}_{R e s_{i}}=S_{i} \pi_{i} \sum_{j \in G} \rho_{i j} \beta_{i j} \frac{I_{R e s_{j}}}{N_{j}}-v I_{\text {Res }_{i}}+\tau \mu I_{S e n_{i}} \\
& -\alpha I_{R e s_{i}}-\gamma I_{R e s_{i}}+\gamma N_{i} \sum_{j \in G} I_{R e s_{j}} \text {. }
\end{aligned}
$$


Sen and Res indicate the antibiotic-sensitive and -resistant N. gonorrhoeae strains, $G=\{L, H\}$ is the set of low and high sexual activity groups and $i \in G$ (Fig 1). Each sexual activity group $N_{i}$ consists of susceptible hosts, $S_{i}$, hosts infected with an antibiotic-sensitive strain, $I_{S^{2} n_{i}}$ and hosts infected with an antibiotic-resistant strain, $I_{\text {Res }_{i}}$. To account for individual heterogeneity in sexual behavior [20], hosts are redistributed to either the same or the other sexual activity group at rate $\gamma$. Redistribution is proportional to the size of the sexual activity group, i.e. hosts from the larger sexual activity group are less likely to change their sexual behavior than hosts from the smaller sexual activity group. Hosts can also leave or enter the population at rate $\alpha$. Susceptible hosts become infected depending on the partner change rate, $\pi_{i}$, the transmission probability per partnership, $\beta_{i j}$, and the sexual mixing matrix, $\rho_{i j}$, which describes how many partnerships are formed within and outside the host's activity group:

$$
\rho_{i j}=\epsilon \delta_{i j}+(1-\epsilon) \frac{\pi_{j} N_{j}}{\sum_{k \in G} \pi_{k} N_{k}},
$$

where $\delta_{i j}=1$ if $i=j$ and $\delta_{i j}=0$ if $i \neq j . \epsilon$ is the sexual mixing coefficient [21]. It ranges from 0 (random or proportionate mixing) to 1 (assortative mixing, i.e. all partnerships are formed with hosts from same group). Hosts infected with an antibiotic-sensitive strain can recover spontaneously at rate $v$ or receive treatment at rate $\tau$. Treatment occurs both when the host seeks treatment for a symptomatic infection or is screened and diagnosed with an asymptomatic infection. Hosts receiving treatment recover at rate $\tau(1-\mu)$ and develop resistance during treatment with probability $\mu$. Hosts infected with an antibiotic-resistant strain can only recover spontaneously at rate $v$. We assumed equal fitness of antibiotic-sensitive and -resistant strains in absence of treatment, i.e. no fitness costs for the antibiotic-resistant strain. We evaluated the impact of fitness costs on the model outcomes in a sensitivity analysis (see S1 Appendix).

Parameters. Model parameters were estimated from sexual behavior data, calibrated through model simulation or informed by literature. The partner change rate and the proportions of the host population in each sexual activity group were estimated from the second

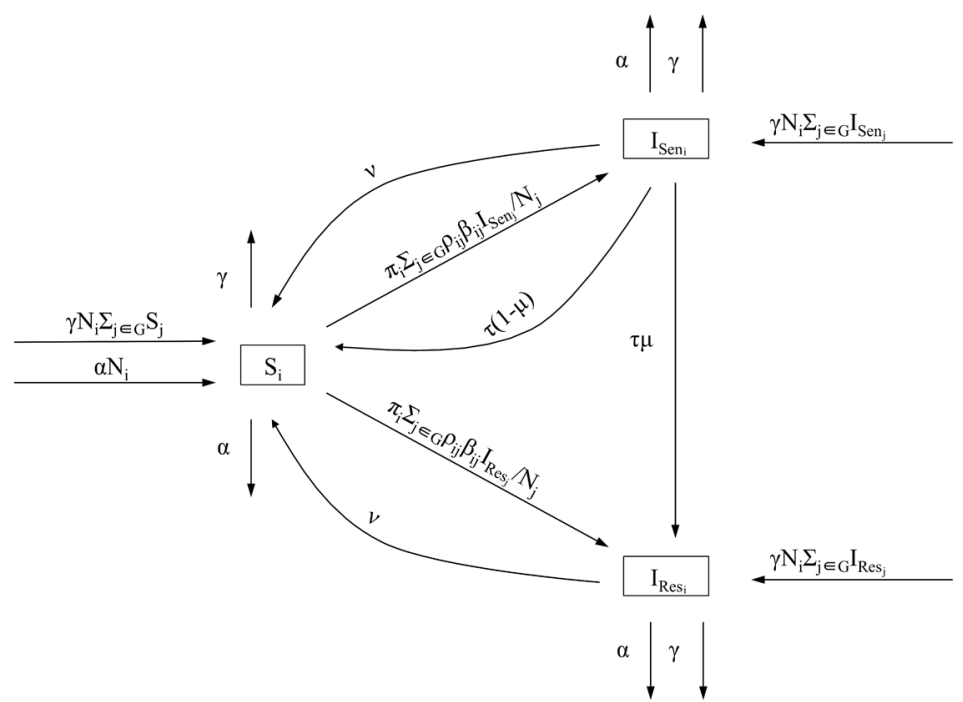

Fig 1. Structure of $\boldsymbol{N}$. gonorrhoeae transmission model. $N_{i}$ sexual activity group $i, S_{i}$ susceptible hosts, $I_{S e n_{i}}$ hosts infected with antibiotic-sensitive strain, $I_{\text {Res }_{i}}$ hosts infected with antibiotic-resistant strain, $\pi_{i}$ partner change rate, $\beta_{i j}$ transmission probability per partnership, $\rho_{i j}$ mixing between and within sexual activity groups, $\tau$ treatment rate, $v$ spontaneous recovery rate, $\mu$ probability of resistance during treatment, $\alpha$ rate of entering and leaving the population, $\gamma$ redistribution rate, $G$ set of low and high sexual activity groups.

doi:10.1371/journal.ppat.1005611.g001 
British National Survey of Sexual Attitudes and Lifestyles (Natsal-2) [22], a population-based cross-sectional survey. For the heterosexual men and women (HMW) model population, we used the number of new heterosexual partners in the last year of all male and female participants between 16-44 years who reported never having had a homosexual partner. For the MSM model population, we used the number of new homosexual partners in the last year of all male participants between 16-44 years who reported ever having had a homosexual partner. For each host population, the number of partners per year was weighted with weights provided in Natsal-2 to adjust for unequal selection probabilities in the survey. We estimated the partner change rate by assuming that the reported numbers of new sexual partners can be described by two Poisson distributions with means $\pi_{L}$ and $\pi_{H}$, weighted by the proportion of individuals in each sexual activity group [23]. For HMW, the sexual partner change rates are $\pi_{L}=0.25 \mathrm{y}^{-1}$ and $\pi_{H}=4.57 \mathrm{y}^{-1}$ with $N_{H}=6.3 \%$ of the population being in the high sexual activity group and $N_{L}=1-N_{H}$. The obtained partner change rates for MSM are $\pi_{L}=0.41 \mathrm{y}^{-1}$ and $\pi_{H}=30.49 \mathrm{y}^{-1}$ with $N_{H}=5.3 \%$ of the population belonging to the high sexual activity group and $N_{L}=1-N_{H}$.

We calibrated the sexual mixing coefficient, $\epsilon$, the fraction of diagnosed and treated infections, $\phi$, the average duration of infection, $D$, and the per partnership transmission probabilities within the low, $\beta_{L L}$, and the high sexual activity group, $\beta_{H H}$, to $N$. gonorrhoeae prevalence and incidence using the following algorithm:

1. Define prior parameter distributions (Table 1).

2. Define the ranges for the expected prevalence and incidence of diagnosed infections (Table 2) of urethral and cervical N. gonorrhoeae infections for HMW, and urethral, rectal and pharyngeal infections for MSM.

3. Randomly draw $10^{7}$ parameters sets from prior distributions.

4. Simulate the transmission model until it approaches a resistance-free $(\mu=0)$ endemic equilibrium using the ordinary differential equation solver runsteady from the $\mathrm{R}$ [19] package rootSolve [24].

5. Select the parameters sets (posterior distributions) that result in prevalences and incidences within the defined range.

Information about parameter estimates for $\mathrm{N}$. gonorrhoeae is scarce, so we chose to use non-informative priors for all parameters except the duration of infection which was informed by Garnett et al. [25]. The ranges for the expected prevalence and incidence of diagnosed

Table 1. Prior distributions and posterior estimates of model parameters.

\begin{tabular}{|c|c|c|c|c|c|c|}
\hline parameter & description & priors & $\mathrm{M}_{M S M}$ & $I_{Q} R_{M S M}$ & $\mathrm{M}_{H M W}$ & $\mathrm{IQR}_{H M W}$ \\
\hline$\epsilon$ & sexual mixing coefficient & $\mathcal{U}(0,1)$ & 0.57 & $0.30-0.80$ & 0.73 & $0.53-0.89$ \\
\hline$\phi$ & fraction of diagnosed and treated infections & $\mathcal{U}(0,1)$ & 0.64 & $0.48-0.81$ & 0.50 & $0.36-0.66$ \\
\hline$D$ & average duration of infection (years) & $\Gamma(2,0.125)$ & 0.19 & $0.14-0.25$ & 0.55 & $0.46-0.66$ \\
\hline$\beta_{L L}$ & transmission probability within low activity group & $\mathcal{U}(0,1)$ & 0.59 & $0.42-0.77$ & 0.87 & $0.79-0.94$ \\
\hline$\beta_{H H}$ & transmission probability within high activity group & $\mathcal{U}\left(0, \beta_{L L}\right)$ & 0.30 & $0.25-0.40$ & 0.72 & $0.63-0.81$ \\
\hline
\end{tabular}

We assumed that the duration of infection is described by a gamma distribution $\Gamma(k, \theta)$ with shape parameter $k=2$ and scale parameter $\theta=0.125 y$ resulting in an average infectious duration of $0.25 \mathrm{y}$. Because highly sexually active hosts have fewer sex acts per partnership, we assumed that the transmission probability within the high activity group cannot be higher than the transmission probability within the low activity group. M and IQR represent the median and interquartile range of the posterior distributions.

doi:10.1371/journal.ppat.1005611.t001 
Table 2. Prevalence and incidence ranges used for model calibration.

\begin{tabular}{lllll}
\hline parameter & infection site & host population & sexual activity group & range \\
\hline prevalence & urethral, cervical & HMW & low & $0-0.38 \%$ \\
prevalence & urethral, cervical & HMW & high & $0.16-100 \%$ \\
prevalence & urethral, cervical & HMW & either & $0.16-0.38 \%$ \\
prevalence & pharyngeal, anal, urethral & MSM & low & $0-2.79 \%$ \\
prevalence & pharyngeal, anal, urethral & MSM & high & $1.19-100 \%$ \\
prevalence & pharyngeal, anal, urethral & MSM & either & $1.19-2.79 \%$ \\
incidence & urethral, cervical & HMW & either & $0.12-0.36 \%$ person $^{-1} \mathrm{y}^{-1}$ \\
incidence & pharyngeal, anal, urethral & MSM & either & $5.88-7.19 \%$ person $^{-1} \mathrm{y}^{-1}$ \\
\hline
\end{tabular}

Prevalence and incidence ranges for HMW were based on the National Health and Nutrition Examination Survey [26] and surveillance data [27], both from CDC. For MSM, prevalence and incidence ranges were based on the Health in Men Study in Australia [28, 29]. The upper and lower bound of the ranges for the low and high sexual activity groups are given by the lower and upper bound of the overall population.

infections in HMW were based on the National Health and Nutrition Examination Survey [26] and surveillance data [27], both from CDC. For MSM, we used data from the Health in Men Study in Australia $[28,29]$. We compared the model predicted prevalence and incidence of diagnosed infections to the prevalence and incidence from data without allowing for resistance in the simulations, because we assumed the data were collected when treatment was mostly effective. We calculated the model incidence of diagnosed and treated infections for sexual activity group $i$ with $\phi S_{i} \pi_{i} \sum_{j \in G} \rho_{i j} \beta_{i j} \frac{I_{\text {Sen }}+I_{\text {Res }}}{N_{j}}$ per year.

We set the rate of entering and leaving the population, $\alpha=\frac{1}{29} \mathrm{y}^{-1}$, because we only considered hosts 16-44 years of age. Since the sexual partner change rates are based on the numbers of new sexual partners within the last year, we assumed that hosts stay on average one year $(\gamma=$ $1 \mathrm{y}^{-1}$ ) in their sexual activity group before they are redistributed to either the same or the other sexual activity group [30]. We do not have information on the probability of resistance during treatment. We set the probability of resistance during treatment to $\mu=10^{-3}$ and performed a sensitivity analysis to assess the impact of $\mu$ on the model outcomes.

The remaining model parameters $\left(\tau, v, \beta_{L H}, \beta_{H L}\right)$ are composites of other parameters (Table 3). Since $D=\frac{1}{v+\tau}$ and $\phi=\frac{\tau}{\tau+v}$, the treatment rate is $\tau=\frac{\phi}{D}$, and the spontaneous recovery rate is $v=\frac{1-\phi}{D} . \beta_{L H}$ and $\beta_{H L}$ are the transmission probabilities per partnership between hosts of the high and low activity groups. We assumed that the between-group transmission probabilities are given by the geometric mean of the within-group transmission probabilities.

Table 3. Composite model parameters.

\begin{tabular}{llr}
\hline parameter & description & formula \\
\hline$\tau$ & treatment rate per year & $\phi / D$ \\
$V$ & spontaneous recovery rate per year & $\frac{1-\phi}{D}$ \\
$\beta_{L H}$ & transmission probability per partnership between low and high sexual activity host & $\sqrt{\beta_{L L} \beta_{H H}}$ \\
$\beta_{H L}$ & transmission probability per partnership between high an low sexual activity host & $\sqrt{\beta_{L L} \beta_{H H}}$ \\
\hline
\end{tabular}

The composite model parameters $\tau$ and $v$ relate to other model parameters with $D=\frac{1}{v+\tau}$ and $\phi=\frac{\tau}{v+\tau}$. We assumed that the transmission probabilities between hosts of different sexual activity groups are given by the geometric mean of the transmission probabilities for hosts within each group.

doi:10.1371/journal.ppat.1005611.t003 


\section{Results}

We fitted a logistic growth model to the proportion of antibiotic-resistant N. gonorrhoeae as observed in the two gonococcal surveillance programs (Fig 2). The proportion of cefiximeresistant $N$. gonorrhoeae in GRASP appears to increase for both HetM and MSM after 2006. Ciprofloxacin-resistant N. gonorrhoeae in HetM and MSM were spreading in all observed host populations after the year 2000. For a given antibiotic and surveillance program, the rates of resistance spread were consistently higher for MSM than for HetM (Table 4). The average rate of resistance spread was $0.53 \mathrm{y}^{-1}$ for HetM and $1.46 \mathrm{y}^{-1}$ for MSM, corresponding to doubling times of $1.3 \mathrm{y}$ (HetM) and $0.5 \mathrm{y}$ (MSM) during the initial exponential growth phase.

Next, we studied the transmission of $N$. gonorrhoeae and the spread of resistance in the dynamic transmission model. We calibrated five model parameters to expected prevalence and incidence in MSM and HMW host populations. The posterior distributions of the parameters were based on 2,779 parameter sets for HMW and 65,699 parameter sets for MSM (Fig 3, Table 1). Distributions of the modeled prevalence and incidence of diagnosed infections after

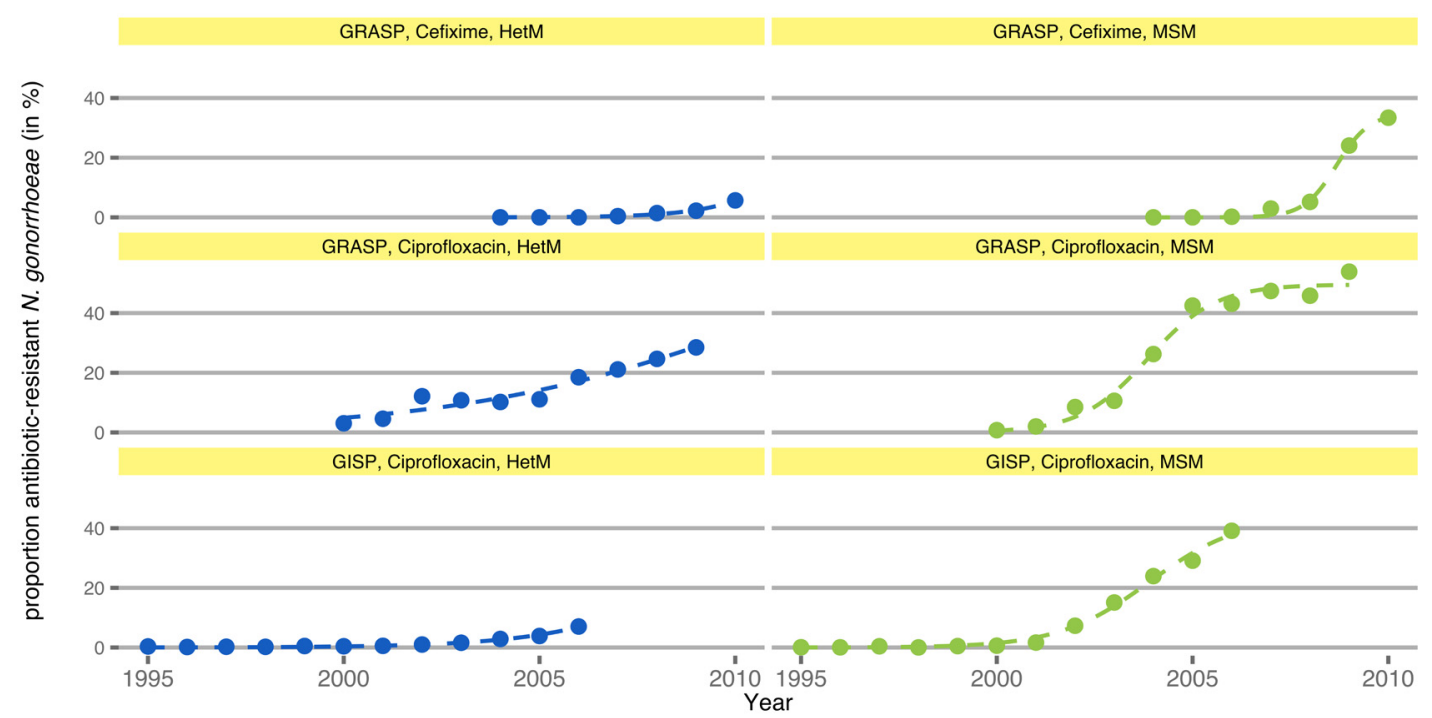

Fig 2. Increase in antibiotic-resistant N.gonorrhoeae. Points show data from antibiotic resistance surveillance programs (GRASP and GISP). Dashed lines indicate the fit of the logistic growth model to the data. For a given antibiotic and surveillance program, the rates of spread in MSM (green) are consistently higher than those in HetM (blue).

doi:10.1371/journal.ppat.1005611.g002

Table 4. Rates of resistance spread in $\mathbf{N}$. gonorrhoeae surveillance programs.

\begin{tabular}{lllll}
\hline program & antibiotic & years & host population & rate (95\% Cl) \\
\hline GRASP & Cefixime & $2004-2010$ & HetM & $0.86(0.73-1.00) \mathrm{y}^{-1}$ \\
GRASP & Cefixime & $2004-2010$ & MSM & $2.38(1.72-3.03) \mathrm{y}^{-1}$ \\
GRASP & Ciprofloxacin & $2000-2009$ & HetM & $0.24(0.03-0.45) \mathrm{y}^{-1}$ \\
GRASP & Ciprofloxacin & $2000-2009$ & MSM & $1.15(0.76-1.54) \mathrm{y}^{-1}$ \\
GISP & Ciprofloxacin & $1995-2006$ & HetM & $0.50(0.45-0.55) \mathrm{y}^{-1}$ \\
GISP & Ciprofloxacin & $1995-2006$ & MSM & $0.86(0.66-1.06) \mathrm{y}^{-1}$ \\
\hline
\end{tabular}

Estimated rates of resistance spread from the Gonococcal Resistance to Antimicrobials Surveillance Programme (GRASP, England and Wales) and from the Gonococcal Isolate Surveillance Project (GISP, USA). Cl: confidence interval.

doi:10.1371/journal.ppat.1005611.t004 
(a)

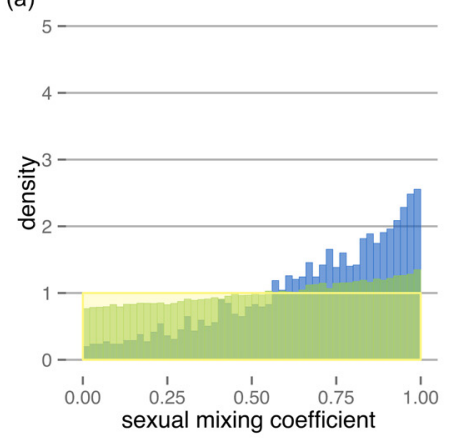

(d)

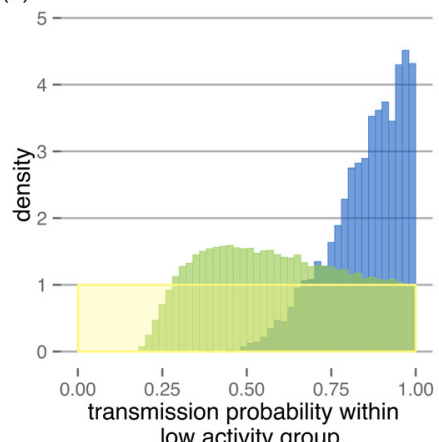

low activity group (b)

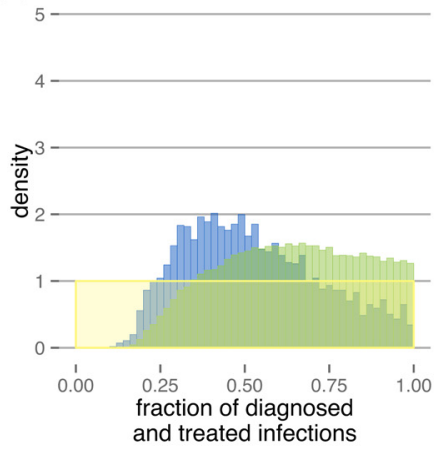

(e)

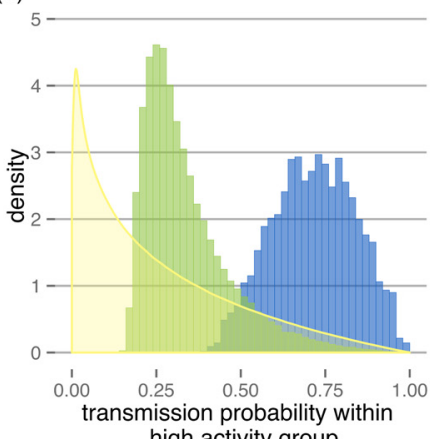

(c)

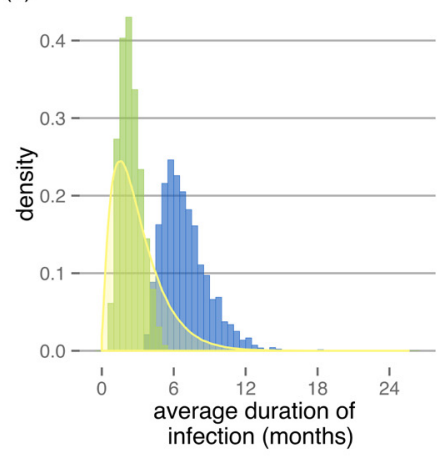

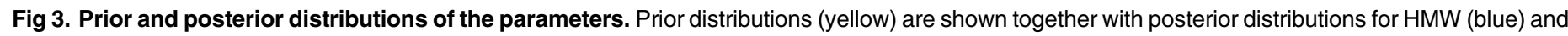

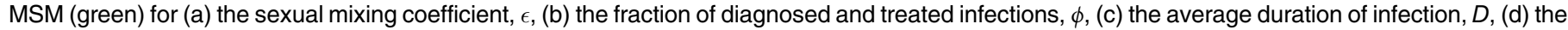
transmission probability within the low activity group, $\beta_{L L}$, and (e) the transmission probability within the high activity group, $\beta_{H H}$.

doi:10.1371/journal.ppat.1005611.g003 


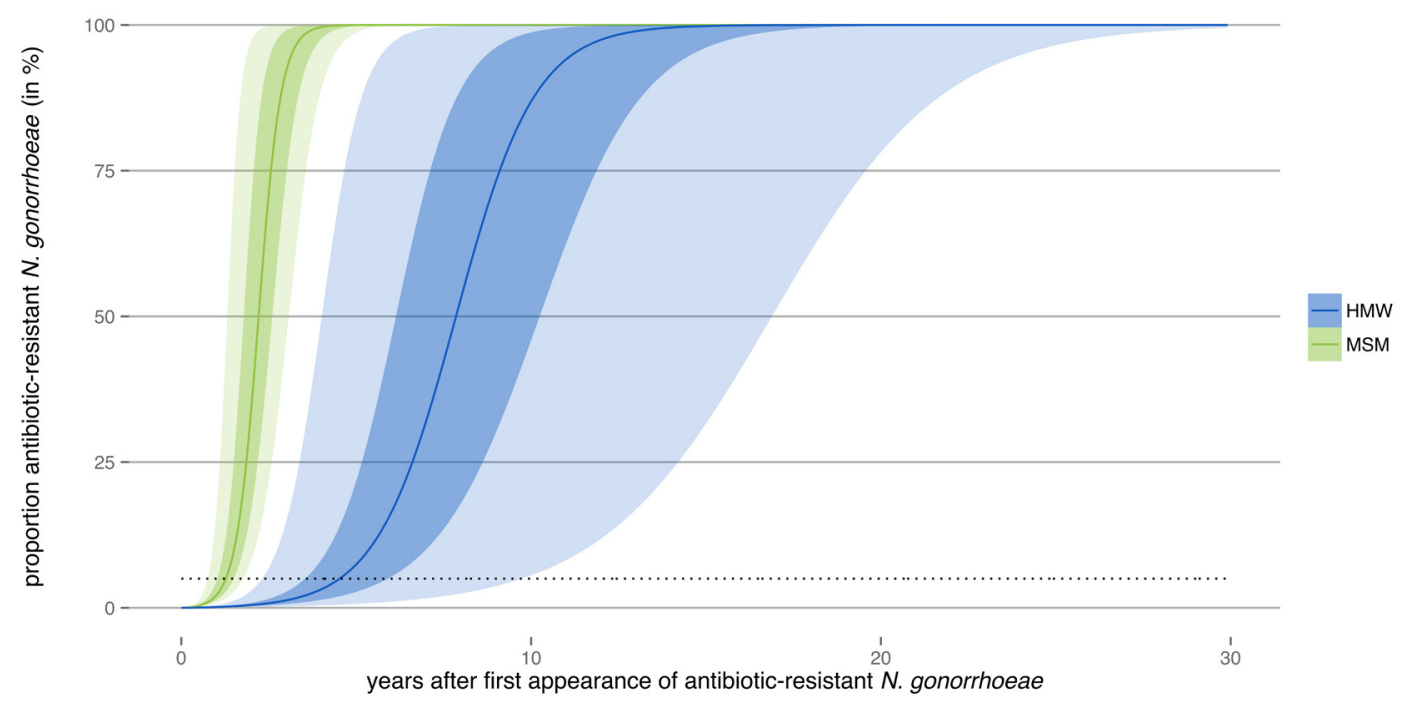

Fig 4. Spread of antibiotic resistance in the transmission model. Ranges indicating $50 \%$ of all simulations are shown in dark color, and ranges indicating $95 \%$ of all simulations are shown in light color. The continuous lines describe the median proportion of antibiotic-resistant $N$. gonorrhoeae for all simulations. The black dotted line indicates the $5 \%$ threshold.

doi:10.1371/journal.ppat.1005611.g004

antibiotic-resistant $N$. gonorrhoeae. The observed distributions of treatment rates from the transmission model hardly overlap between HMW and MSM (Fig 5). The median treatment rates, i.e. the approximated median rates of resistance spread in the transmission model are $3.12 \mathrm{y}^{-1}$ (MSM) and $0.88 \mathrm{y}^{-1}$ (HMW).

We tested whether changes in the probability of resistance during treatment, $\mu$, and fitness costs in the antibiotic-resistant strain alter the model outcomes. Higher probabilities of resistance during treatment accelerate the establishment of antibiotic-resistant N. gonorrhoeae in the population and hence reduce the time until 5\% resistance is reached (S3 Fig). Higher probabilities of resistance during treatment, however, do not affect rates of spread, unless the

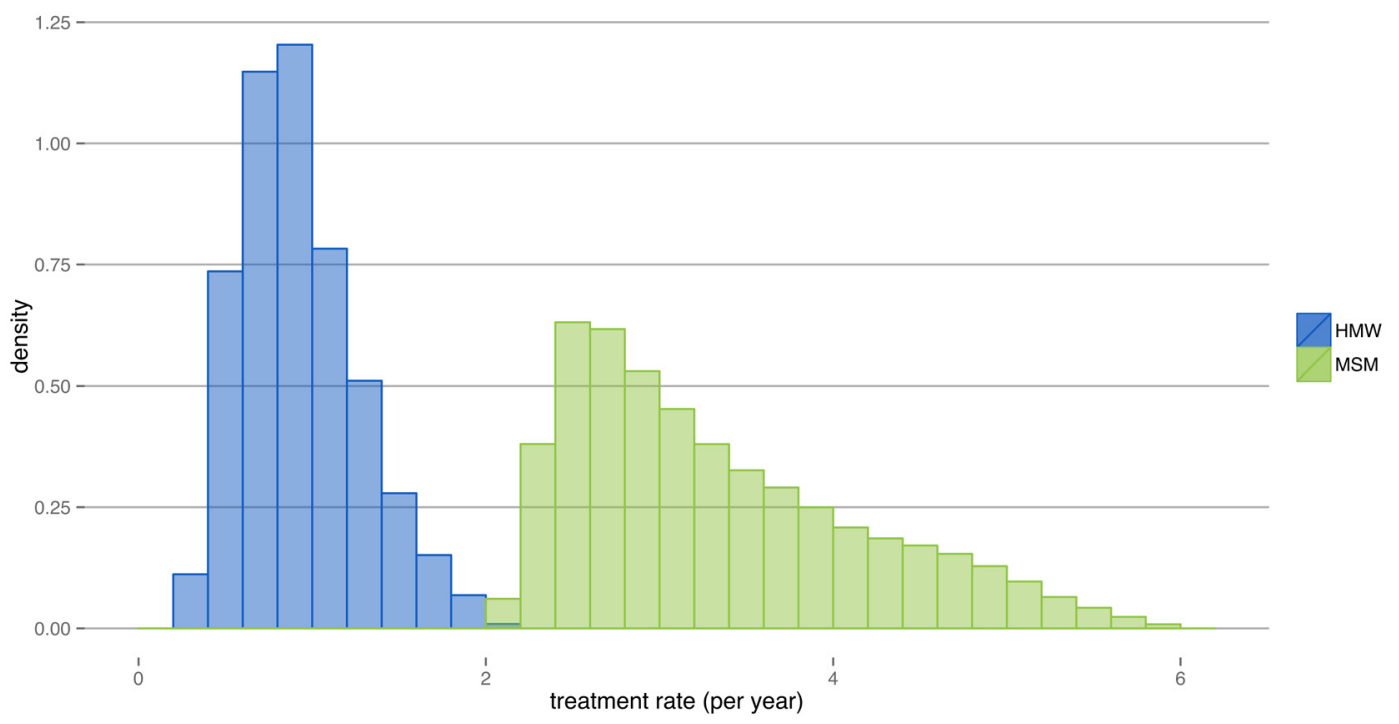

Fig 5. Distribution of treatment rates in HMW and MSM. Treatment rates closely approximate the rates of resistance spread. The median treatment rate was $0.88 \mathrm{y}^{-1}$ in HMW and $3.12 \mathrm{y}^{-1}$ in MSM.

doi:10.1371/journal.ppat.1005611.g005 
probability of resistance during treatment is unrealistically high (10\%) (S4 Fig). Fitness costs in the antibiotic-resistant strain result in rates of resistance spread that are lower than the treatment rate $\tau$ (Fig. B in S1 Appendix). Fitness costs that reduce the transmission probability per partnership, $\beta_{i j}$, have a stronger effect than fitness costs that reduce the duration of infection. The effects of fitness costs are independent of the sexual partner change rate, $\pi_{i}$, and $\beta_{i j}$ if they affect the duration of infection, but can vary with $\pi_{i}$ and $\beta_{i j}$ if they affect the transmission probability per partnership (Fig. C in S1 Appendix). While high fitness costs can prevent the spread of antibiotic-resistant strains (Fig. A in S1 Appendix), fitness costs between $0 \%-10 \%$ have only small effects on the rates of resistance spread (Fig. B in S1 Appendix).

\section{Discussion}

In this study, we quantified the rate at which antibiotic-resistant $N$. gonorrhoeae spread in heterosexual and MSM populations. We used data from two different surveillance programs and estimated that the proportion of ciprofloxacin- and cefixime-resistant $N$. gonorrhoeae doubles on average every $1.3 \mathrm{y}$ in HetM and $0.5 \mathrm{y}$ in MSM. The faster spread of antibiotic-resistant $N$. gonorrhoeae in MSM than in heterosexual hosts was corroborated using a dynamic transmission model, which was calibrated to observed prevalence and incidence rates. The model allowed us to identify the higher treatment rates in MSM, compared with heterosexual hosts, as the major driver for the faster spread of antibiotic-resistant $N$. gonorrhoeae.

To our knowledge, this is the first study to have analyzed and interpreted N. gonorrhoeae antibiotic resistance surveillance data in a dynamic and quantitative manner. The transmission model was parameterized using sexual behavior data for HMW and MSM from Natsal-2 [22], a large probability sample survey of sexual behavior. Calibrating the model to observed prevalence and incidence rates allowed us to use largely uninformative priors for the model parameters. The calibration makes our model more robust to changes in parameters than using fixed parameter values, especially since for $N$. gonorrhoeae available parameter values are very uncertain [31]. It also allowed us to rely on few assumptions about the natural history of N. gonorrhoeae infection.

The limitations to our study need to be taken into consideration when interpreting the findings. First, we used data from different sources, although all were collected in high income countries. The antibiotic resistance surveillance data are from programs in England and Wales and the USA. The mathematical transmission model was parameterized using British sexual behavior data [22] and calibrated to prevalence and incidence rates from the USA (HMW) [26, 27] and Australia (MSM) [28, 29]. For simplicity, we modeled the heterosexual and MSM host populations separately although there is some mixing between them. We assumed the sexual behavior of heterosexual men and women to be the same and pooled their behavioral data. Second, we assumed complete resistance against the antibiotic, i.e. $100 \%$ treatment failure. We further assumed that treatment of the sensitive strain is $100 \%$ efficacious. Both assumptions might explain why antibiotic-resistant $N$. gonorrhoeae spread at somewhat higher rates in the dynamic transmission model than estimated from data. Third, we restricted our model to resistance to one antibiotic with no alternative treatment or interventions. This is why we observe complete replacement of the antibiotic-sensitive strain in the model, a phenomenon that has not been observed in surveillance data. Fourth, resistance in our model is treated as a generic trait, but it likely depends on the underlying molecular mechanisms and possibly the genetic background of the N. gonorrhoeae strain. Different resistance mechanisms might explain some of the differences in the rates of resistance spread between the model and the different antibiotics from the surveillance data. Fifth, we did not include co- and superinfection with antibioticsensitive and -resistant $N$. gonorrhoeae strains. Since genetic typing provides evidence for 
mixed infections [32], it is worth speculating how they would affect the rate of spread from the transmission model. If antibiotic-sensitive and -resistant strains co-existed in a host and acted independently, we would not expect significant effects on the rate of spread. In contrast, if there was competition between the two strains within a host, the rate of spread would increase if the antibiotic-resistant strain outcompetes the -sensitive strain, and decrease otherwise. Sixth, we do not consider importation of resistance from another population. For example, importation of resistance from other countries might play a particularly important role during the early phase of resistance spread, when stochastic events can lead to extinction of the antibiotic-resistant strain. We expect that a high rate of importation of antibiotic resistance shortens the time to reach $5 \%$ resistance drastically, but that once the resistant strain is established in the population, importation hardly affects the rate of resistance spread. Finally, we assumed that the transmission probabilities per partnership and the durations of infection in the model represent average values for $N$. gonorrhoeae infections at different infection sites (urethral, pharyngeal, anal, cervical).

The estimated posterior distributions of the parameters fit within the range of previously used values, and provide some insights into sexual mixing and the natural history of N. gonorrhoeae. The sexual mixing coefficient tends to be assortative for both HMW and MSM host populations in our model. Quantifying the degree of sexual mixing is difficult and largely depends on the study population, but our finding is consistent with other studies indicating assortative sexual mixing in the general population [30,33]. The posterior estimates of the fraction of diagnosed and treated infections are consistent with the notion that a large proportion of $N$. gonorrhoeae infections are symptomatic, and that this proportion is expected to be higher in men than in women [34-36]. The average duration of infection was the only parameter with an informative prior, but we found marked differences between the duration of infection in HMW (6.6 months) and MSM (2.3 months). Per sex act transmission probabilities are generally considered to be lower from women to men than vice versa [37-39]. In our model, the median of the transmission probability per partnership was lower in MSM hosts than in HMW for both sexual activity groups. This could be explained by different numbers of sex acts per partnership in the two populations. The low transmission probability within the highly active MSM group (median: 30\%) could reflect a single or a small number of sex acts per partnership. In contrast, the high transmission probability for HMW within the low sexual activity group (median: 87\%) could be a result of a larger number of sex acts per partnership in those individuals. Furthermore, condom use is more frequent in MSM than in HMW [22], which could explain part of the observed differences in transmission probabilities.

Our study found that the treatment rate is the driving force of resistance spread. Xiridou et al. [13] found that resistance could spread faster when the treatment rate was higher, but they did not identify the treatment as the major driver of resistance spread. Chan et al. [12] found that focusing treatment on the core group leads to a faster rebound to pre-treatment prevalence than equal treatment of the entire host population. Unfortunately, our findings cannot be compared with Chan et al. because they do not report the proportion of antibiotic-resistant N. gonorrhoeae.

It was shown previously that treatment is the main selective force acting on resistance evolution due to the selective advantage to the resistant pathogen [40,41]. We now expand this concept by showing that, assuming no fitness costs, treatment rates determine the rates of resistance spread even when the host populations has a heterogeneous contact structure. The intuitive argument that a faster spread of an infection, due to a higher number of sexual partners, will result in a faster spread of resistance does not hold. Instead, the proportion of resistant infections spreads equally in host populations with different number of partners as long as they receive treatment at the same rate and there are no fitness costs associated with the 
transmission probability per partnership. For N. gonorrhoeae, this insight challenges the current management strategy that aims to lower the overall burden of infection by expanding screening and treatment of hosts $[9,10]$. As soon as antibiotic-resistant pathogens are frequent enough to evade stochastic extinction, expanded treatment will foster their spread and increase the burden of $N$. gonorrhoeae. Additionally, we show that fitness costs can decelerate or even prevent the spread of antibiotic-resistant $N$. gonorrhoeae strains. Fitness costs therefore might explain why highly resistant strains, such as the ceftriaxone-resistant $N$. gonorrhoeae strain H041, do not spread in the host population after their first detection [42]. Our findings also show that bridging between the HetM and the MSM host populations might not have been necessary for cefixime-resistance to spread in the HetM population after 2010 [5]. It is likely that cefixime-resistant $N$. gonorrhoeae had already been present in the HetM population but were spreading at a lower rate than in the MSM population.

The results of our study will be useful for future N. gonorrhoeae research and for guiding treatment recommendations. The $N$. gonorrhoeae transmission model describes observed prevalence and incidence rates well and can reconstruct the spread of antibiotic-resistant $N$. gonorrhoeae. Estimating rates of resistance spread is useful for projecting future resistance levels and the expected time it will take until a certain threshold in the proportion of antibiotic-resistant $N$. gonorrhoeae is reached. Until now, treatment recommendations for N. gonorrhoeae are subject to change when $5 \%$ of $N$. gonorrhoeae isolates show resistance against a given antibiotic [6]. Our study shows the importance of the rate of spread: a level of $5 \%$ resistance results in a marginal increase to $8 \%$ in the following year if resistance spreads logistically at rate $0.53 \mathrm{y}^{-1}$ (HetM mean estimate from Table 4), but reaches $18 \%$ in the next year if resistance spreads at rate $1.46 \mathrm{y}^{-1}$ (MSM mean estimate from Table 4). Public health authorities could use surveillance data and adapt thresholds for treatment recommendation change to specific host populations using the method we describe. Our study challenges the currently prevailing notion that more screening and treatment will limit the spread of $N$. gonorrhoeae, as higher treatment rates will ultimately result in faster spread of antibiotic resistance. Future treatment recommendations for N. gonorrhoeae should carefully balance prevention of N. gonorrhoeae infection and avoidance of the spread of resistance.

\section{Supporting Information}

S1 Table. Digitized data from the Gonococcal Resistance to Antimicrobials Surveillance Programme (GRASP). Data for heterosexual men (HetM) and men who have sex with men (MSM).

S2 Table. Digitized data from the Gonococcal Isolate Surveillance Project (GISP). Data for men who have sex with women (MSW) and men who have sex with men (MSM). We used the MSW data as heterosexual men (HetM) data. (PDF)

S3 Table. Prevalence and incidence of diagnosed and treated infections after model calibration.

(PDF)

S1 Fig. Posterior distributions of (a) prevalence and (b) incidence of diagnosed and treated infections for MSM.

(TIFF) 
S2 Fig. Posterior distributions of (a) prevalence and (b) incidence of diagnosed and treated infections for HMW.

(TIFF)

S3 Fig. Sensitivity of time to $5 \%$ resistance towards changes in the probability of resistance during treatment, $\boldsymbol{\mu}$. The time to $5 \%$ resistance of both MSM (green) and HMW (blue) are sensitive towards $\mu$. Lower and upper bound of the box indicate the first and third quartiles, bar in the box indicates median, whiskers span 1.5 times IQR. Outliers are shown in orange and are outside 1.5 times IQR.

(TIFF)

S4 Fig. Sensitivity of rate of spread towards changes in the probability of resistance during treatment, $\boldsymbol{\mu}$. The rates of spread of both MSM (green) and HMW (blue) are only sensitive towards $\mu$ when $\mu$ is unrealistically high. Lower and upper bound of the box indicate the first and third quartiles, bar in the box indicates median, whiskers span 1.5 times IQR. Outliers are shown in orange and are outside 1.5 times IQR.

(TIFF)

S1 Appendix. Fitness costs and spread of resistance.

(PDF)

\section{Acknowledgments}

We would like to thank Sandro Gsteiger and Denise Kühnert for advice on parameter calibration, and Fengyi Jin and Andrew Grulich for providing the MSM prevalence and incidence data from the Health in Men Study.

\section{Author Contributions}

Conceived and designed the experiments: SMF SB NL CLA. Performed the experiments: SMF. Analyzed the data: SMF SB NL CLA. Wrote the paper: SMF SB NL CLA.

\section{References}

1. Unemo M, Shafer WM. Antimicrobial Resistance in Neisseria gonorrhoeae in the 21st Century: Past, Evolution, and Future. Clin Microbiol Rev. 2014 jul; 27(3):587-613. Available from: http://www.ncbi. nlm.nih.gov/pubmed/24982323. doi: 10.1128/CMR.00010-14 PMID: 24982323

2. Centers for Disease Control and Prevention. Sexually Transmitted Disease Surveillance 2012. Atlanta: U.S. Department of Health and Human Services; 2014.

3. Public Health England. GRASP 2013 Report The Gonococcal Resistance to Antimicrobials Surveillance Programme (England and Wales); 2014.

4. Davies S, Fowler T, Watson J, Livermore D, Walker D. Annual Report of the Chief Medical Officer: infection and the rise of antimicrobial resistance. Lancet. 2013; 381:1606-1609. Available from: http:// www.thelancet.com/journals/lancet/article/PIIS0140-6736(13)60604-2/abstract. doi: 10.1016/S01406736(13)60604-2 PMID: 23489756

5. Ison CA, Town K, Obi C, Chisholm S, Hughes G, Livermore D, et al. Decreased susceptibility to cephalosporins among gonococci: data from the Gonococcal Resistance to Antimicrobials Surveillance Programme (GRASP) in England and Wales, 2007-2011. Lancet Infect Dis. 2013 sep; 13(9):762-8. Available from: http://www.ncbi.nlm.nih.gov/pubmed/23764300. doi: 10.1016/S1473-3099(13)70143-9 PMID: 23764300

6. Tapsall J, World Health Organization. Antimicrobial resistance in Neisseria gonorrhoeae; 2001. Available from: http://www.who.int/entity/csr/resources/publications/drugresist/Neisseria_gonorrhoeae.pdf.

7. Bignell C, Unemo M. 2012 European Guideline on the Diagnosis and Treatment of Gonorrhoea in Adults. Int J STD AIDS. 2013 feb; 24(2):85-92. Available from: http://www.ncbi.nlm.nih.gov/pubmed/ 24400344. doi: 10.1177/0956462412472837 PMID: 24400344 
8. Kirkcaldy RD, Weinstock HS, Moore PC, Philip SS, Wiesenfeld HC, Papp JR, et al. The Efficacy and Safety of Gentamicin Plus Azithromycin and Gemifloxacin Plus Azithromycin as Treatment of Uncomplicated Gonorrhea. Clin Infect Dis. 2014 oct; 59(8):1083-91. Available from: http://www.ncbi.nlm.nih. gov/pubmed/25031289. doi: 10.1093/cid/ciu521 PMID: 25031289

9. Campos-Outcalt D. CDC update on gonorrhea: Expand treatment to limit resistance. J Fam Practice. 2011; 60(12):736-739.

10. Centers for Disease Control and Prevention. Cephalosporin-resistant Neisseria gonorrhoeae Public Health Response Plan; 2012. Available from: http://www.cdc.gov/std/treatment/ceph-rresponseplanjuly30-2012.pdf.

11. Yorke J, Hethcote H, Nold A. Dynamics and Control of the Transmission of Gonorrhea. Sex Transm Dis. 1978; 5(2):51-56. Available from: http://journals.Iww.com/stdjournal/Abstract/1978/04000/ Dynamics_and_Control_of_the_Transmission_of.3.aspx.PMID: 10328031

12. Chan $\mathrm{CH}, \mathrm{McC}$ abe $\mathrm{CJ}$, Fisman DN. Core groups, antimicrobial resistance and rebound in gonorrhoea in North America. Sex Transm Infect. 2012 apr; 88(3):200-4. Available from: http://www.ncbi.nlm.nih. gov/pubmed/22169277. doi: 10.1136/sextrans-2011-050049 PMID: 22169277

13. Xiridou M, Soetens LC, Koedijk FDH, van der Sande MAB, Wallinga J. Public health measures to control the spread of antimicrobial resistance in Neisseria gonorrhoeae in men who have sex with men. Epidemiol Infect. 2015 oct; 143(8):1575-1584. Available from: http://www.ncbi.nlm.nih.gov/pubmed/ 25275435. doi: 10.1017/S0950268814002519 PMID: 25275435

14. Hui BB, Ryder N, Su JY, Ward J, Chen MY, Donovan B, et al. Exploring the Benefits of Molecular Testing for Gonorrhoea Antibiotic Resistance Surveillance in Remote Settings. PloS One. 2015; 10(7): e0133202. doi: 10.1371/journal.pone.0133202 PMID: 26181042

15. Health Protection Agency. GRASP 2011 Report: The Gonococcal Resistance to Antimicrobials Surveillance Programme. 2012;p. 12. Available from: http://webarchive.nationalarchives.gov.uk/ 20140714084352/http://www.hpa.org.uk/webc/HPAwebFile/HPAweb_C/1317136030908.

16. Health Protection Agency. GRASP 2011 Report: The Gonococcal Resistance to Antimicrobials Surveillance Programme. 2012;p. 14. Available from: http://webarchive.nationalarchives.gov.uk/ 20140714084352/http://www.hpa.org.uk/webc/HPAwebFile/HPAweb_C/1317136030908.

17. Centers for Disease Control and Prevention. Sexually Transmitted Disease Surveillance 2011. 2012;p. 54. Available from: http://www.cdc.gov/std/stats11/surv2011.pdf.

18. Plot Digitizer 2.6.6. 2014;Available from: http://plotdigitizer.sourceforge.net/.

19. R Core Team. R: A Language and Environment for Statistical Computing. Vienna, Austria; 2015. Available from: http://www.r-project.org/.

20. Romero-Severson EO, Alam SJ, Volz EM, Koopman JS. Heterogeneity in Number and Type of Sexual Contacts in a Gay Urban Cohort. Stat Commun Infect Dis. 2012; 4(1). Available from: http://www. pubmedcentral.nih.gov/articlerender.fcgi?artid=3639013\&tool=pmcentrez\&rendertype=abstract.

21. Garnett GP, Swinton J, Brunham RC, Anderson RM. Gonococcal infection, infertility, and population growth: II. The influence of heterogeneity in sexual behaviour. IMA J Math Appl Med Biol. 1992; 9 (2):127-144. doi: 10.1093/imammb/9.2.127 PMID: 1517674

22. Johnson AM, Mercer CH, Erens B, Copas AJ, McManus S, Wellings K, et al. Sexual behaviour in Britain: Partnerships, practices, and HIV risk behaviours. Lancet. 2001; 358(9296):1835-1842. doi: 10. 1016/S0140-6736(01)06883-0 PMID: 11741621

23. Althaus CL, Heijne JCM, Herzog SA, Roellin A, Low N. Individual and Population Level Effects of Partner Notification for Chlamydia trachomatis. PloS One. 2012 jan; 7(12):e51438. Available from: http:// www.pubmedcentral.nih.gov/articlerender.fcgi?artid=3520891\&tool=pmcentrez\&rendertype=abstract. doi: 10.1371/journal.pone.0051438 PMID: 23251534

24. Soetaert, K. rootSolve: Nonlinear root finding, equilibrium and steady-state analysis of ordinary differential equations; 2009. Available from: https://cran.r-project.org/web/packages/rootSolve/.

25. Garnett GP, Mertz KJ, Finelli L, Levine WC, Louis ME St. The transmission dynamics of gonorrhoea: modelling the reported behaviour of infected patients from Newark, New Jersey. Philos Trans R Soc Lond B Biol Sci. 1999 apr; 354(1384):787-97. Available from: http://www.pubmedcentral.nih.gov/ articlerender.fcgi?artid=1692556\&tool=pmcentrez\&rendertype=abstract. doi: 10.1098/rstb.1999.0431 PMID: 10365404

26. Datta SD, Sternberg M, Johnson RE, Berman S, Papp JR, McQuillan G, et al. Gonorrhea and Chlamydia in the United States among Persons 14 to 39 Years of Age, 1999 to 2002. Ann Intern Med. 2007; 147(2):89-96. doi: 10.7326/0003-4819-147-2-200707170-00007 PMID: 17638719

27. Centers for Disease Control and Prevention. Sexually Transmitted Disease Surveillance 2013. Atlanta: U.S. Department of Health and Human Services; 2014. 
28. Jin F, Prestage GP, Mao L, Kippax SC, Pell CM, Donovan B, et al. Incidence and risk factors for urethral and anal gonorrhoea and chlamydia in a cohort of HIV-negative homosexual men: the Health in Men Study. Sex Transm Infect. 2007; 83(2):113-119. doi: 10.1136/sti.2006.021915 PMID: 17005541

29. Jin F. Personal Communication. 2015 may.

30. Althaus CL, Choisy M, Alizon S, CSF group. Number of sex acts matters for heterosexual transmission and control of Chlamydia trachomatis. PeerJ PrePrints. 2015; 3:e1164. Available from: https://dx.doi. org/10.7287/peerj.preprints.940v1.

31. Grad YH, Goldstein E, Lipsitch M, White PJ. Improving Control of Antibiotic-Resistant Gonorrhea by Integrating Research Agendas Across Disciplines: Key Questions Arising From Mathematical Modeling. J Infect Dis. 2016; 213(6):883-890. Available from: http://jid. oxfordjournals.org/content/213/6/883. doi: 10.1093/infdis/jiv517 PMID: 26518045

32. Lynn F, Hobbs MM, Zenilman JM, Behets FMTF, Van Damme K, Rasamindrakotroka A, et al. Genetic typing of the porin protein of Neisseria gonorrhoeae from clinical noncultured samples for strain characterization and identification of mixed gonococcal infections. J Clin Microbiol. 2005; 43(1):368-375. doi: 10.1128/JCM.43.1.368-375.2005 PMID: 15634996

33. Renton A, Whitaker L, Ison C, Wadsworth J, Harris JRW. Estimating the sexual mixing patterns in the general population from those in people acquiring gonorrhoea infection: theoretical foundation and empirical findings. J Epidemiol Community Health. 1995 apr; 49(2):205-213. Available from: http://jech. bmj.com/cgi/doi/10.1136/jech.49.2.205. doi: 10.1136/jech.49.2.205 PMID: 7798052

34. Farley TA, Cohen DA, Elkins W. Asymptomatic sexually transmitted diseases: the case for screening. Prev Med. 2003; 36(4):502-509. Available from: http://www.sciencedirect.com/science/article/pii/ S0091743502000580. doi: 10.1016/S0091-7435(02)00058-0 PMID: 12649059

35. Buimer M, van Doornum GJ, Ching S, Peerbooms PG, Plier PK, Ram D, et al. Detection of Chlamydia trachomatis and Neisseria gonorrhoeae by ligase chain reaction-based assays with clinical specimens from various sites: implications for diagnostic testing and screening. J Clin Microbiol. 1996; 34 (10):2395-400. Available from: http://www.pubmedcentral.nih.gov/articlerender.fcgi?artid= 229278\&tool=pmcentrez\&rendertype=abstract. PMID: 8880487

36. Chen MI, Ghani AC, Edmunds WJ. A metapopulation modelling framework for gonorrhoea and other sexually transmitted infections in heterosexual populations. J R Soc Interface. 2009 sep; 6(38):775791. Available from: http://rsif.royalsocietypublishing.org/cgi/doi/10.1098/rsif.2008.0394. doi: 10.1098/ rsif.2008.0394 PMID: 18986961

37. Hethcote H, Yorke J. Gonorrhea Transmission Dynamics and Control. Lecture Notes in Bioinformatics. 1984; 56. doi: 10.1007/978-3-662-07544-9

38. Swinton J, Garnett GP, Brunham RC, Anderson RM. Gonococcal infection, infertility, and population growth: I. Endemic states in behaviourally homogeneous growing populations. IMA J Math Appl Med Biol. 1992; 9(2):107-126. doi: 10.1093/imammb/9.2.107 PMID: 1517673

39. Bracher M, Watkins S, Santow G. "Moving" and Marrying: Modelling HIV Infection among Newly-weds in Malawi. Demogr Res. 2003 sep; Special 1:207-246. Available from: http://www.demographicresearch.org/special/1/7/. doi: 10.4054/DemRes.2003.S1.7

40. Bonhoeffer S, Lipsitch M, Levin BR. Evaluating treatment protocols to prevent antibiotic resistance. Proc Natl Acad Sci USA. 1997 oct; 94(22):12106-11. Available from: http://www.ncbi.nlm.nih.gov/ pubmed/9342370. doi: 10.1073/pnas.94.22.12106 PMID: 9342370

41. Huijben S, Bell AS, Sim DG, Tomasello D, Mideo N, Day T, et al. Aggressive Chemotherapy and the Selection of Drug Resistant Pathogens. PLoS Pathog. 2013 sep; 9(9):e1003578. doi: 10.1371/journal. ppat.1003578 PMID: 24068922

42. Shimuta K, Unemo M, Nakayama SI, Morita-Ishihara T, Dorin M, Kawahata T, et al. Antimicrobial Resistance and Molecular Typing of Neisseria gonorrhoeae Isolates in Kyoto and Osaka, Japan, 2010 to 2012: Intensified Surveillance after Identification of the First Strain (H041) with High-Level Ceftriaxone Resistance. Antimicrob Agents Chemother. 2013 nov; 57(11):5225-32. Available from: http://www. ncbi.nlm.nih.gov/pubmed/23939890 http://www.pubmedcentral.nih.gov/articlerender.fcgi?artid= PMC3811299. doi: 10.1128/AAC.01295-13 PMID: 23939890 